\title{
Convergência entre as Taxas de Mortalidade Infantil e os Índices de Desenvolvimento Humano no Brasil no período de 2000 a 2010
}

\author{
The Convergence betwixt Infant Mortality Rate and Human Development Index \\ in Brazil between 2000 to 2010 \\ La convergence entre les Taxes de la Mortalité Infantile et les Taux de \\ Developpement Humain au Brésil dans la période allant de 2000 à 2010
}

Convergencia entre las Tasas de Mortalidad Infantil y los Índices de Desarrollo Humano en Brasil en el período 2000 a 2010

\author{
Paulo Cezar Rodrigues Martins ${ }^{1}$ \\ Elenir Rose Jardim Cury Pontes ${ }^{1}$ \\ Leandro Tsuneki Higa² \\ Recebido em 03/04/2017; revisado e aprovado em 04/06/2017; aceito em 12/06/2017 \\ DOI: http://dx.doi.org/10.20435/inter.v19i2.1552
}

\begin{abstract}
Resumo: Este estudo tem como objetivo verificar a convergência entre a diminuição da Taxa de Mortalidade Infantil e o aumento do Índice de Desenvolvimento Humano Municipal nas Grandes Regiões e Unidades da Federação no Brasil no período de 2000 a 2010. O tipo de estudo adotado foi o ecológico. Conclui-se que houve redução das Taxas de Mortalidade Infantil e aumento do Índice de Desenvolvimento Humano Municipal, de forma não homogênea nas áreas de estudo.
\end{abstract}

Palavras-chave: mortalidade infantil; desenvolvimento humano; estudo ecológico.

Abstract: This study has as objective to verify the convergence betwixt Infant Mortality Rate decreasement and Human Development Index Municipal increasement in Big Regions and Federation Units in Brazil between 2000 to 2010. The type of study adopted is ecological. It is drawn to a conclusion that the Infant Mortality Rate's reduction and the Human Development Index Municipal's increase, happened in a non-homogeneous form in the areas of study.

Keywords: infant mortality; human development index; ecological study.

Résumé: Cette étude a comme but de vérifier la convergence entre la diminution de la Taxe de Mortalité Infantile et l'augmentation du taux de Développement Humain Municipal dans les Grandes Régions et les Unités de la Fédération au Brésil dans la période allant de 2000 à 2010. Le genre d'étude adopté c'est l'écologique. La conclusion c'est qu'il a eu réduction des Taxes de la Mortalité Infantile et l'augmentation du taux du Développement Humain Municipal, d'une façon pas homogène dans les terrains d'étude.

Mots-clés: mortalite infantile; developpement humain; étude écologique.

Resumen: Este estudio tiene como objetivo verificar la convergencia entre la disminución de la Tasa de Mortalidad Infantil y el aumento del Índice de Desarrollo Humano Municipal en las Grandes Regiones y Unidades de la Federación en Brasil en el período de 2000 a 2010. El tipo de estudio adoptado fue el Ecológico. Se concluye que hubo reducción de las Tasas de Mortalidad Infantil y aumento del Índice de Desarrollo Humano Municipal, de forma no homogénea en las áreas de estudio.

Palabras clave: mortalidad infantil; desarrollo humano; estudio ecológico.

\section{INTRODUÇÃO}

A Taxa de Mortalidade Infantil (TMI), frequência de óbitos de crianças menores de um ano de idade por mil nascidos vivos, é um dos indicadores mais sensíveis da situação de saúde e condições de vida da população. Uma criança com menos de um ano de idade é bastante vul-

\footnotetext{
${ }^{1}$ Universidade Federal de Mato Grosso do Sul (UFMS), Campo Grande, Mato Grosso do Sul, Brasil.

2 Instituto Brasileiro de Geografia e Estatística (IBGE), Campo Grande, Mato Grosso do Sul, Brasil.
} 
nerável a fatores determinantes na gestação, no parto e pós-parto. Vários sistemas fisiológicos estão em desenvolvimento no primeiro ano de vida, entre os quais, o imunológico que requer atenção nutricional e cuidados com agentes patológicos ambientais (MEDRONHO et al., 2009). A TMI estima a probabilidade (risco) de morte de uma criança não atingir seu primeiro ano de vida (REDE INTERAGENCIAL DE INFORMAÇÕES PARA A SAÚDE [RIPSA], 2008).

Os óbitos de crianças menores de um ano de idade representam mais de $85 \%$ dos óbitos de menores de cinco anos no Brasil. A TMl é o segundo indicador dos Objetivos de Desenvolvimento do Milênio-4. A meta que era atingir 15,7\%o em 2015 foi alcançada em 2011 com 15,3 óbitos de menores de um ano por mil nascidos vivos (INSTITUTO DE PESQUISA ECONÔMICA APLICADA [IPEA], 2014).

As estimativas de óbitos diários de menores de um ano no mundo são de 15.087, dos quais 14.890 são em países considerados em desenvolvimento, e 197, em países mais desenvolvidos. Ou seja, de cada mil óbitos de menores de um ano que ocorrem por dia, cerca de 99 são em países em desenvolvimento (POPULATION REFERENCE BUREAU [PRB], 2014). Existem enormes diferenças nas TMIs entre os países, em extremos encontramos TMIs abaixo de 2\%o (Luxemburgo, Islândia e Finlândia) e acima de 80\%o (Angola, Serra Leoa e Somália) (THE WORLD BANK GROUP, 2016).

Um dos efeitos da queda da mortalidade de menores de um ano é o aumento da expectativa de vida da população. De acordo com a Tábua de Mortalidade 2015, a expectativa de vida dos brasileiros atingiu 75,5 anos e a TMI foi de 13,8 óbitos de menores de um ano por mil nascidos vivos. Em 1940, esses números eram respectivamente: 45,5 anos e 146,6\%o (IBGE, 2016).

Estudos de análises individuais e ecológicos associam os óbitos infantis às condições socioeconômicas, as mais frequentemente citadas são: disponibilidade de saneamento básico, escolaridade da mãe, raça/cor, média de moradores por domicílio, residência em áreas urbano/ rural e nível de renda (KAEMPFFER; MEDINA, 2006; SCHELL et al., 2007).

Novos conceitos de bem-estar humano têm sido discutidos pelos países como forma de expressar o desenvolvimento humano. O Estado de Madhya Pradesh, na Índia, adota a mortalidade infantil como proxy de uma vida longeva e saudável na elaboração do IDH (PROGRAMA DAS NAÇÕES UNIDAS PARA O DESENVOLVIMENTO [PNUD], 2015).

Composto por três dimensões - uma vida longeva e saudável mensurada pela esperança de vida ao nascer, aptidão de lograr conhecimento medida pela média de anos de estudo e possibilidade de atingir um padrão de vida digno, medido pelo rendimento nacional bruto per capita - foi elaborado o Índice de Desenvolvimento Humano (IDH). Diante disso, permite-se uma abordagem mais global em relação a outras abordagens de caráter estritamente da riqueza. O Brasil ocupa a 75a (IDH = 0,755) posição, em 2014, no grupo de desenvolvimento humano elevado. Mesmo com avanços no índice, o Brasil na posição fica atrás de: Argentina (40a), Chile

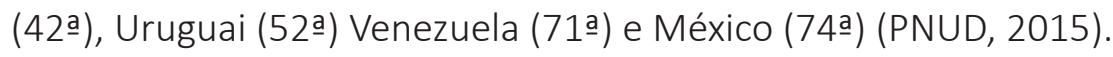

Em 1998, o Brasil foi precursor em adequar e elaborar um IDH subnacional a nível municipal. O Índice de Desenvolvimento Humano Municipal (IDHM) difunde o conceito de desenvolvimento focado nas pessoas, deixando de ter um olhar apenas no crescimento econômico (PNUD, 2013).

A acessibilidade, abrangência geográfica, relação implícita do indivíduo com o ambiente e a comparabilidade fizeram com que este estudo elegesse as Taxas de Mortalidade Infantil (TMI) e o IDHM como medidas capazes de traduzir o desenvolvimento humano. Logo, este estudo procurou descrever a evolução das TMIs e o IDHM no Brasil, Grandes Regiões e Unidades da 
Federação no período de 2000 a 2010, e verificar a convergência entre a diminuição da taxa de mortalidade infantil e o aumento do IDHM das áreas estudadas.

\section{METODOLOGIA}

O presente trabalho foi classificado como ecológico. Esse tipo de estudo permitiu conhecer a distribuição da mortalidade infantil- que expressa a qualidade do saneamento básico, dos serviços de saúde e da assistência materno-infantil, em relação ao desenvolvimento humano nas áreas estudadas.

As áreas eleitas para este estudo foram as Unidades da Federação e Grandes Regiões brasileiras. Foram utilizadas medidas individuais de mortalidade infantil dentro de cada área que foram sintetizadas em uma medida agregada, a Taxa de Mortalidade Infantil (TMI), e uma medida global, o IDHM.

Os dados referentes às Taxas de Mortalidade Infantil foram extraídos dos Indicadores Básicos para a Saúde (IDB) (RIPSA, 2012) para os anos de 2000 e 2010. Essa base de dados de indicadores é produto da Rede Interagencial de Informações para a Saúde (Ripsa), que tem como objetivo subsidiar com informações relevantes os processos de formulação, gestão e avaliação de políticas e ações públicas de importância estratégica para o sistema de saúde brasileiro.

O indicador C.1- Taxa de Mortalidade Infantil refere-se ao número de óbitos de menores de um ano de idade, por mil nascidos vivos, na população residente em determinado espaço geográfico, no ano considerado. As taxas foram elaboradas a partir de dados do Sistema de Informações sobre Nascidos Vivos (SINASC) e do Sistema de Informações sobre Mortalidade (SIM), ambos da Secretaria de Vigilância em Saúde do Ministério da Saúde (SVS-MS), posteriormente foram corrigidos por fatores obtidos na Pesquisa de Busca Ativa realizada na Amazônia Legal e no Nordeste (RIPSA, 2012).

O Índice de Desenvolvimento Humano Municipal agrega indicadores de três dimensões do desenvolvimento humano: longevidade, educação e renda. O índice varia de 0 a 1 , quanto mais próximo de 1 , maior é o desenvolvimento humano. As dimensões do IDH Global são as mesmas do IDHM, porém a composição dos indicadores de cada uma delas difere. Outro aspecto a ser considerado é a fonte de dados, enquanto no IDHM os dados são extraídos dos Censos Demográficos, o IDH global busca os dados nas Nações Unidas, Organização das Nações Unidas para Educação, Ciência e Cultura. Diante disso, esses índices não são comparáveis entre si.

Os resultados do IDHM das Unidades da Federação e Brasil foram transcritos da plataforma do Atlas do Desenvolvimento Humano no Brasil, enquanto o IDHM das Grandes Regiões do Brasil tiveram como fonte a publicação "Desenvolvimento Humano nas Macro Regiões Brasileiras" (PNUD, 2016).

O IDHM-Brasil é construído a partir de indicadores provenientes das informações dos Censos Demográficos, ou seja, dos residentes nos domicílios. Esse procedimento é diferente de se calcular a média aritmética simples dos IDHMs dos 5565 municípios do País. Nesse caso, estaríamos considerando municípios de forma que todos os municípios entrariam com o mesmo peso no cálculo, independente do porte populacional. Outra forma seria o cálculo do IDHM do País por meio da média aritmética ponderada, tendo como peso o número de habitantes. Esse procedimento se aproximaria do valor obtido pelo método adotado para o IDHM do Brasil. Outro aspecto a ser considerado é o subíndice "Esperança de vida ao nascer", pois teria seu cálculo comprometido, tendo em vista que ele é elaborado a partir de técnicas demográficas indiretas 
e também influenciado pela estrutura etária da população. Para as Unidades da Federação e Grandes Regiões, a metodologia é a mesma.

Segundo a metodologia do Atlas do Desenvolvimento Humano no Brasil, as faixas do IDHM estão classificadas em: muito baixo (0 a 0,499); baixo (0,500 a 0,599); médio $(0,600$ a 0,699); alto $(0,700$ a 0,799$)$; muito alto $(0,800$ a 1,00$)$.

Para analisar se houve tendência à redução ou não das diferenças de TMI e IDHMs entre as Unidades da Federação (UFs) estudadas no período avaliado, foi realizada a análise de convergência $(\sigma)$. A convergência $(\sigma)$ caracteriza-se por uma redução da dispersão dos valores dos indicadores ao longo do tempo, o que significaria que a TMI e o IDHM das diferentes regiões tende a se aproximar de sua média. Assim, a convergência $(\sigma)$ foi testada pela análise do coeficiente de variação (CV), dado pela razão entre o desvio-padrão e a média aritmética dos indicadores em termos relativos (\%). Quanto menor for o valor do coeficiente de variação, mais homogêneos serão os dados, ou seja, menor será a dispersão em torno da média.

O cálculo do Coeficiente de variação é dado por meio da fórmula:

$$
\mathrm{CV}=\frac{\text { desvio-padrão }}{\text { média }} \cdot 100
$$

Os resultados deste estudo estão apresentados por meio de tabelas, mapas e gráficos.

\section{MORTALIDADE INFANTIL: BRASIL, GRANDES REGIÕES E UNIDADES DA FEDERAÇÃO}

As Taxas de Mortalidade Infantil no Brasil, observadas no período 2000/2010, apresentaram uma redução significativa. Esse fato foi verificado também nas Grandes Regiões e nas Unidades da Federação. A TMI, em 2000, no Brasil foi de 26,1 óbitos de menores de um ano para cada mil nascidos vivos, essa taxa passou para 16 óbitos em 2010. Uma redução de 38,7\% no período (Tabela 1). Em nível global, a TMI em 2015 é menor do que a metade da referida em 1990, tendo descido de noventa para 43 mortes por mil nascidos vivos, ou de cerca de 12,7 milhões para seis milhões em termos absolutos (PNUD, 2015).

Quando se observa as TMls segundo as grandes regiões, em 2000, apenas a Região Sul indicava a TMI abaixo de vinte óbitos para cada mil nascidos vivos. Enquanto nas demais Regiões se observaram valores acima de vinte. Já em 2010, apenas a Região Norte, apesar da redução de 36\%, ainda manteve a TMI acima de $20 \%$. As demais Regiões apresentaram a TMI abaixo de vinte óbitos para cada mil nascidos vivos. Dentre as Regiões, a que teve maior redução no período 2000/2010 foi a do Nordeste, cerca de 47\%. A menor TMI em 2010 foi observada na Região Sul (11,58\%o) (Tabela 1). 
Tabela 1 - Taxas de Mortalidade Infantil (TMI) e Índice de Desenvolvimento Humano Municipal (IDHM), segundo Brasil, Grandes regiões e Unidades da Federação- 2000/2010

\begin{tabular}{|c|c|c|c|c|}
\hline $\begin{array}{c}\text { Grandes Regiões e } \\
\text { Unidades da Federação }\end{array}$ & TMI \%о $2000^{(1)}$ & TMI \%о $2010^{(1)}$ & IDHM $2000^{(2)}$ & IDHM $2010^{(2)}$ \\
\hline Brasil & 26,10 & 16,00 & 0,612 & 0,727 \\
\hline Norte & 32,80 & 20,97 & 0,527 & 0,667 \\
\hline Acre & 29,20 & 20,42 & 0,517 & 0,663 \\
\hline Amazonas & 34,80 & 20,57 & 0,515 & 0,674 \\
\hline Amapá & 32,87 & 25,41 & 0,577 & 0,708 \\
\hline Pará & 32,30 & 21,46 & 0,518 & 0,646 \\
\hline Rondônia & 31,94 & 18,92 & 0,537 & 0,690 \\
\hline Roraima & 22,22 & 18,00 & 0,598 & 0,707 \\
\hline Tocantins & 36,92 & 20,45 & 0,525 & 0,699 \\
\hline Nordeste & 35,93 & 19,09 & 0,516 & 0,663 \\
\hline Alagoas & 37,70 & 18,58 & 0,471 & 0,631 \\
\hline Bahia & 34,56 & 21,04 & 0,512 & 0,660 \\
\hline Ceará & 36,79 & 16,23 & 0,541 & 0,682 \\
\hline Maranhão & 36,80 & 21,88 & 0,476 & 0,639 \\
\hline Paraíba & 39,16 & 18,19 & 0,506 & 0,658 \\
\hline Pernambuco & 33,96 & 17,04 & 0,544 & 0,673 \\
\hline Piauí & 37,79 & 20,70 & 0,484 & 0,646 \\
\hline Rio grande do Norte & 34,47 & 17,23 & 0,552 & 0,684 \\
\hline Sergipe & 37,65 & 18,17 & 0,518 & 0,665 \\
\hline Centro-Oeste & 22,32 & 15,93 & 0,639 & 0,757 \\
\hline Distrito federal & 15,27 & 12,16 & 0,725 & 0,824 \\
\hline Goiás & 21,21 & 15,89 & 0,615 & 0,735 \\
\hline Mato Grosso do Sul & 24,37 & 15,44 & 0,613 & 0,729 \\
\hline Mato Grosso & 29,38 & 19,55 & 0,601 & 0,725 \\
\hline Sudeste & 20,09 & 13,43 & 0,676 & 0,766 \\
\hline Espírito Santo & 18,13 & 11,91 & 0,640 & 0,740 \\
\hline Minas Gerais & 25,73 & 16,16 & 0,624 & 0,731 \\
\hline Rio de Janeiro & 20,50 & 14,31 & 0,664 & 0,761 \\
\hline São Paulo & 17,37 & 12,04 & 0,702 & 0,783 \\
\hline Sul & 16,92 & 11,58 & 0,660 & 0,754 \\
\hline Paraná & 19,02 & 12,03 & 0,650 & 0,749 \\
\hline Rio Grande do Sul & 15,33 & 11,33 & 0,664 & 0,746 \\
\hline Santa Catarina & 15,90 & 11,18 & 0,674 & 0,774 \\
\hline
\end{tabular}

Fonte: (1) RIPSA (2012); (2) PNUD (2013; 2016).

As TMIs no Brasil tiveram redução substancial nas últimas décadas, de 5,5\% ao ano nas décadas de 1980 e 1990 e de 4,4\% ao ano desde 2000, chegando a vinte óbitos por mil nascidos vivos em 2008. As desigualdades regionais em relação à mortalidade infantil foram reduzidas. A acessibilidade aos serviços de saúde materna e infantil tiveram incrementos, passando para uma cobertura quase universal (VICTORA et al., 2011).

Em 2000, dentre as 27 Unidades da Federação (UF), 21 apresentaram TMI acima de 20\% e seis abaixo de 20\%o. Dez anos depois, dezenove UFs tinham uma TMI abaixo de 20\%, e somente oito acima de $20 \%$ (Tabela 1 e Figura 1 ). 
Países em desenvolvimento e com desigualdades socioeconômicas devem monitorar a evolução do objetivo quatro dos ODMs em escala estadual e municipal, verificando os hiatos de desigualdades existentes a fim de promover um melhor direcionamento das políticas de saúde e ações entre setores públicos (SOUSA; HILL; DAL POZ, 2010).

Os resultados da TMI Brasil revelam grandes distâncias entre os valores. Municípios com piores indicadores socioeconômicos apresentam maiores riscos de uma criança morrer antes de completar o primeiro ano de vida. Os resultados observados demonstram associação das condições de vida da população com os locais de residência, evidenciando as disparidades regionais (FISCHER et al., 2007).
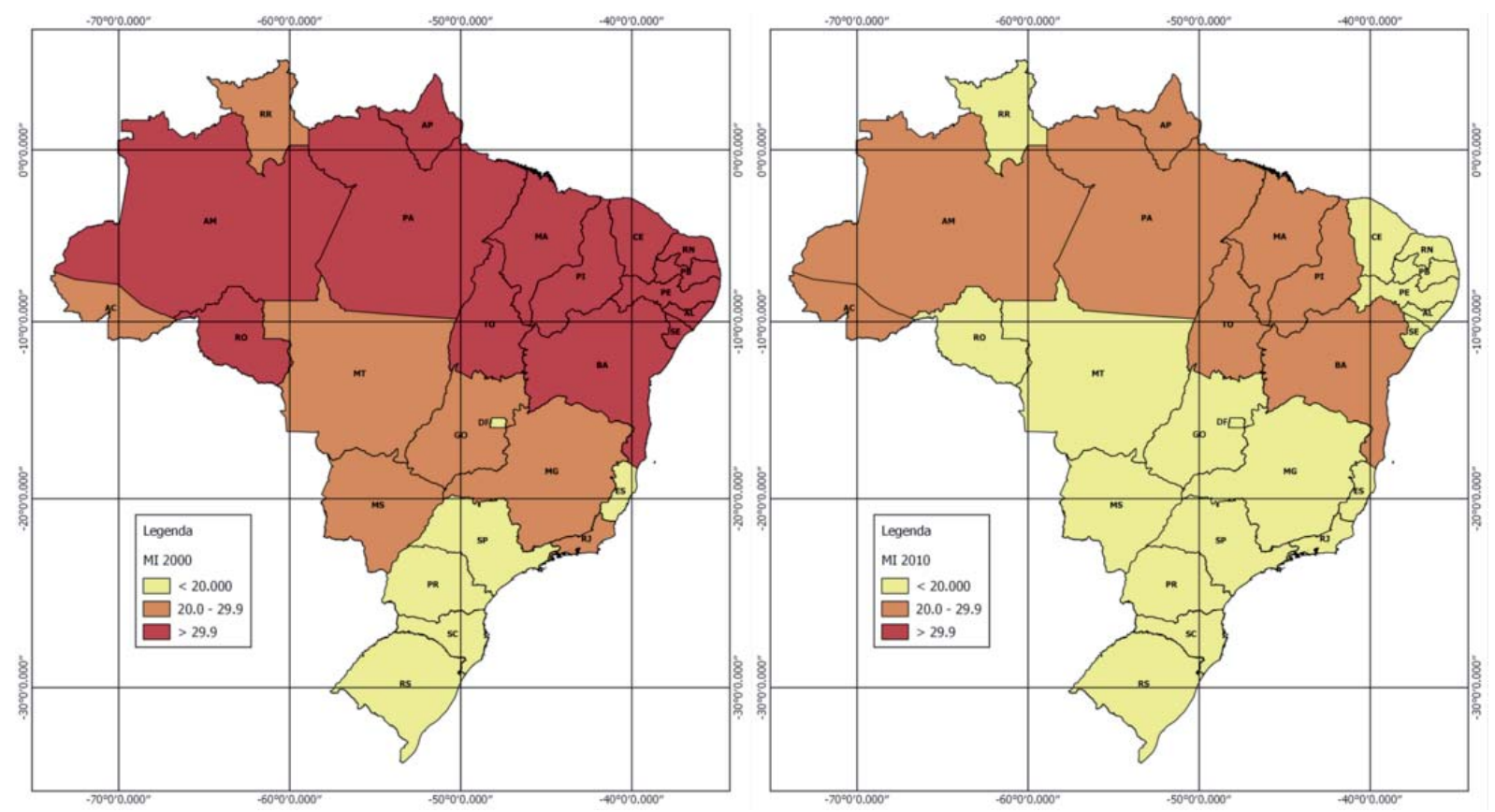

Figura 1 - Taxa de Mortalidade Infantil (TMI), por faixas, segundo as Unidades da Federação2000/2010

A menor TMI encontrada em 2000 foi de 15,27 óbitos em menores de um ano de idade no Distrito Federal e a maior foi de 39,16\% na Paraíba. Em 2010, o Estado de Santa Catarina registrou a menor TMI $(11,18 \%)$ e a maior foi observada no Amapá $(25,41 \%)$. Cabe ressaltar que a diferença entre o maior e o menor valor encontrado em 2000 era cerca de 24 óbitos, já essa diferença, em 2010, diminui para 14 óbitos de menores de um ano. A redução entre o maior e menor valor da TMI sugere um avanço na redução das desigualdades entre as UFs (Tabela 1).

O Estado do Ceará foi o que obteve maior redução $(55,88 \%)$ na TMI no período em análise, de 36,79\%o óbitos para 16,23\%o. Quando observamos as dez maiores reduções da TMI, sete são da Região Nordeste (CE, PB, SE, AL, RN, PE e PI) e três ( $A M, R O$ e MA) da Região Norte. Esses Estados demonstraram maior sensibilidade às políticas e ações voltadas para minimizar as desigualdades socioeconômicas que atingiam suas populações (Tabela 1 e Figura 1).

Os Estados da Região Nordeste foram os que tiveram os maiores percentuais de redução da TMI. Maior ênfase verificou-se no Estado de Alagoas, onde, em 2000, a TMI era 37,7\%o e, em 2013, passou para 15,2\%o, ou seja, uma redução de 60\%. Dentre as UFs, observou-se a diminui- 
ção entre valores máximos e mínimos, em 1990 era de 76 pontos e apenas 11,5 pontos no ano de 2013. Entre os maiores desafios para redução da TMI na Região Norte, que apresentaram as maiores TMIs, estão as características geográficas e demográficas (BRASIL, 2015).

Dos resultados encontrados na Região Norte em 2010, apenas dois Estados (RO e RR) tiveram a TMI abaixo de 20\%. Nos demais Estados (AC, AM, AP, PA e TO), foram observadas taxas superiores a $20 \%$. Destaca-se o Amapá com a maior TMI $(25,41 \%$ ) de todos os estados brasileiros. Dentre as dez maiores taxas dos estados brasileiros, seis são da Região Norte (Tabela 1 e Figura 1).

A maior TMI verificada na Região Nordeste, em 2010, foi no Estado do Maranhão (21,88\%o) e a menor no Ceará (16,23\%o), que, por sua vez, também registrou a décima primeira menor TMI das UFs. Conforme descrito anteriormente, a Região Nordeste foi a que teve mais estados com maiores reduções da TMI no período de 2000 a 2010 (Tabela 1 e Figura 1).

Os Estados do Nordeste, Rio Grande do Norte, Bahia, Ceará e Alagoas foram os que tiveram maior redução da TMI na década de 1990. Reduções que tiveram como principal determinante o maior acesso à educação e à rede de água, aumento da renda e queda das taxas de fecundidade (SOUSA; LEITE FILHO, 2008).

Na Região Centro-Oeste, em 2010, todas as UFs apresentaram a TMI abaixo de 20\%. O Distrito Federal teve a menor TMI (12,16\%) da região, fato que ocorre desde 2000 (15,27\%o). A maior redução $(36,64 \%)$ observada na região foi no Estado de Mato Grosso do Sul, pois, em 2000, tinha uma TMI de 24,37\% e, dez anos mais tarde, registrou uma TMI de 15,44\%o (Tabela 1 e Figura 1).

As TMls observadas nos Estados da Região Sudeste, em 2010, situam-se abaixo de 20\%. Destaque vai para o Estado do Espírito Santo, que tinha uma TMI de 18,13\%o e que foi reduzida para 11,91\%, sendo a terceira menor taxa do País no ano de 2010 dentre as UFs (Tabela 1 e Figura 1).

Segundo o IBGE, a TMI no Brasil, em 2014, foi de 14,4 mortes por mil nascidos vivos. Porém, as diferenças regionais ainda persistiam. Observa-se que as regiões Norte $(18,6)$, Nordeste $(18,4)$ e Centro-Oeste $(15,2)$ possuem valores acima da média, enquanto Sudeste $(11,1)$ e Sul $(10,1)$ apresentam valores inferiores. A maior TMI coube ao Amapá $(23,7)$, enquanto a menor, ao Espírito Santo $(9,6)$ (IBGE, 2015).

Desde 2000, os Estados integrantes da Região Sul já apresentavam TMIs abaixo de 20\%. As taxas observadas nos Estados estão bem próximas entre si, indicando uma homogeneidade nos indicadores. Santa Catarina detém a menor TMI (11,18\%) do Brasil, seguida pelo Rio Grande do Sul (11,33\%) (Figura 1 e 2 ). 


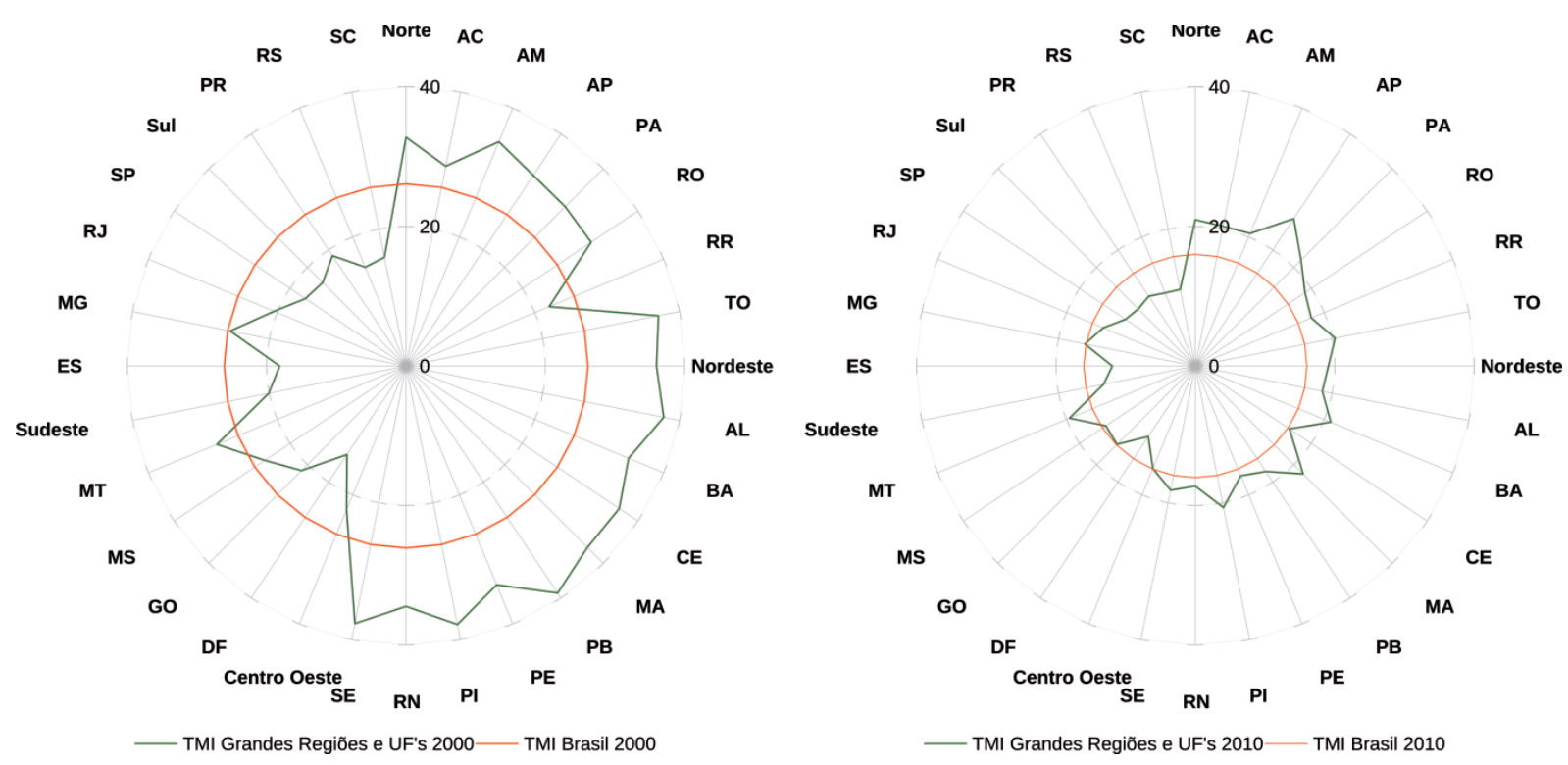

Figura 2 - Distribuição da TMI, segundo as Unidades da Federação, Grandes Regiões em relação à taxa do Brasil- 2000/2010

Na Figura 2, verifica-se que, em 2010, todos os estados da Região Norte e Nordeste mais os Estados de Mato Grosso e Minas Gerais apresentavam as TMIs acima do resultado do Brasil.

\section{IDHM: BRASIL, GRANDES REGIÕES E UNIDADES DA FEDERAÇÃO}

O desenvolvimento humano no Brasil passou de médio (0,612 em 2000) para alto $(0,727$ em 2010), uma variação de $18,7 \%$ no período (Tabela 1 ). Dentre as dimensões do IDHM, a longevidade $(0,816$ em 2010) foi o mais alto subíndice do IDHM do Brasil, refletindo a queda dos níveis de fecundidade e da mortalidade infantil.

Observa-se que houve melhora do IDHM em todas as Regiões Brasileiras. Em 2000, verificou-se que duas Regiões com IDHM baixo (Norte e Nordeste), passando para médio em 2010. Enquanto as regiões Centro-Oeste, Sudeste e Sul de médio passaram para alto no mesmo período. Em 2010, apesar das Regiões Norte e Nordeste apresentarem IDHM menores que as demais regiões, no período de 2000 a 2010, os maiores avanços no desenvolvimento humano foram observados nessas regiões (Tabela 1 ).

Uma das limitações da análise do IDHM segundo as Grandes Regiões relaciona-se às diferenças internas, induzindo muitas vezes à falsa impressão de que os resultados dos índices dos municípios e das Unidades da Federação são homogêneos.

O IDHM, quando analisado de uma forma macro, pode ocultar bolsões de vulnerabilidade. O Programa Comunidade Solidária, a exemplo, excluiu do programa os municípios de porte médio da Região Sudeste, tendo em vista que os resultados do IDHM eram maiores que os da Região Norte e Nordeste. Se fossem utilizados indicadores de áreas submunicipais (bairros, áreas de ponderação e setores censitários), estes poderiam identificar áreas prioritárias tais quais as das Regiões Norte e Nordeste (JANUZZI, 2005).

Em 2000, 11,11\% das UFs tinham desenvolvimento humano considerado muito baixo, $48,15 \%$ baixo, 33,33\% médio e 7,41\% alto. No ano de 2010, a maior concentração $(51,85 \%)$ das UFs está na faixa do desenvolvimento médio e 44,44\% alto. Na série do IDHM, o Distrito Federal foi a primeira UF a atingir a classificação de desenvolvimento muito alto (Figura 3). 


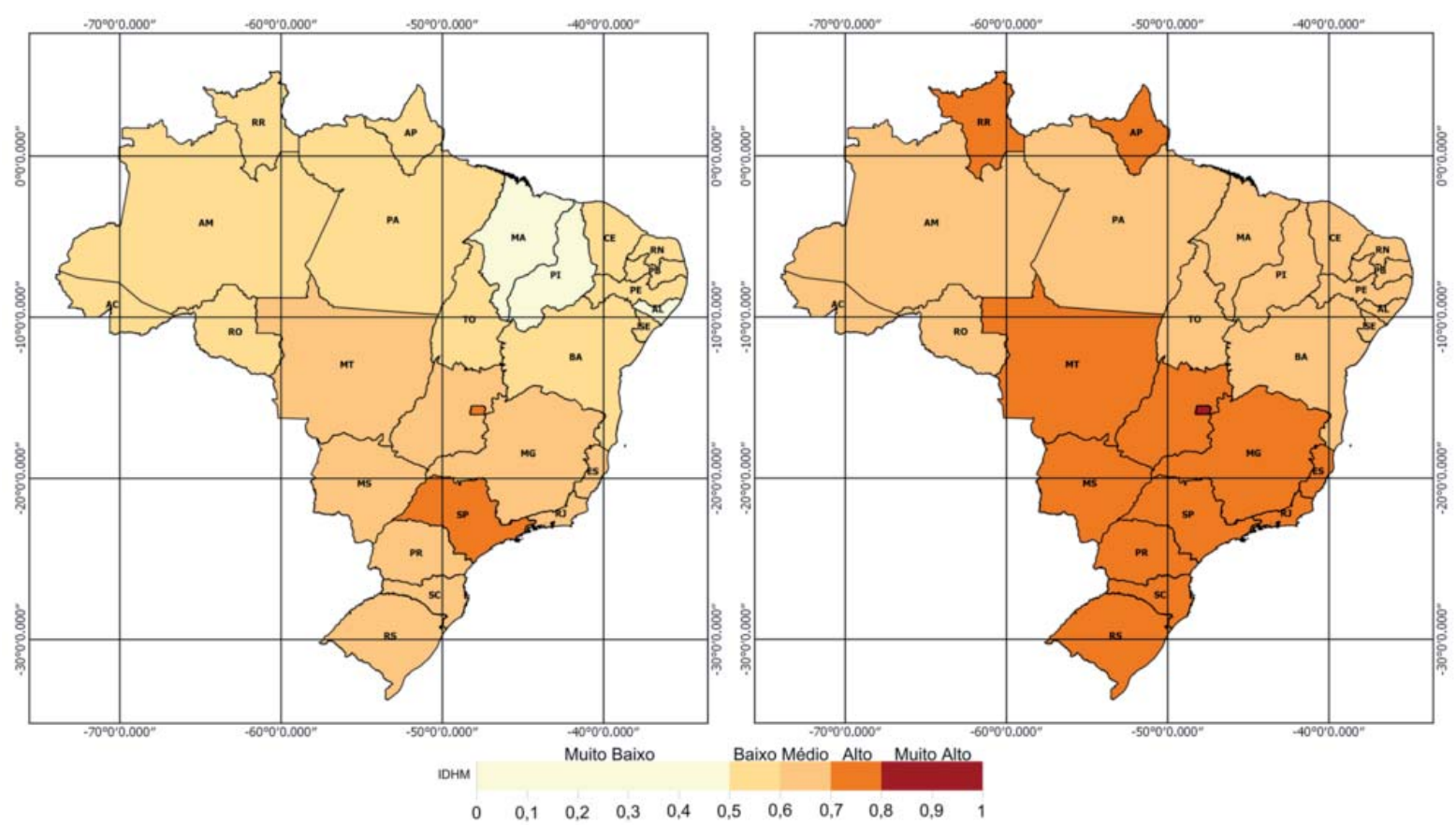

Figura 3 - IDHM, por faixas, segundo as Unidades da Federação- 2000/2010

Na Região Norte, em 2000, os sete Estados (AP, RR, TO, RO, AM, AC e PA) apresentaram o IDHM considerado baixo. Dez anos após, cinco deles passaram ao IDHM médio (AC, AM, PA, RO e TO) e dois obtiveram a classificação de alto desenvolvimento humano (AP e TO) (Tabela 1 e Figura 3).

As diferenças regionais do IDHM no Brasil são notórias nos últimos resultados de 2010. A Região Norte e Nordeste, mesmo com a ascensão nos níveis de renda, apresenta 61,3\% de seus municípios classificados como baixo desenvolvimento humano. Apesar da evolução da qualidade de vida nos últimos anos, o IDHM da Região Nordeste situa-se abaixo da média nacional e das demais macrorregiões. A proximidade geográfica entre as regiões pode promover a interação espacial e influenciar os resultados do IDHM via fluxos de renda, conhecimento, indivíduos, bens e serviços (LINS et al., 2015).

Em 2000, apenas três Estados do Brasil tiveram o IDHM na faixa de muito baixo desenvolvimento humano, todos localizados na Região Nordeste (AL, MA e PI), os demais estados da Região obtiveram IDHM baixo (CE, BA, PB, PE, RN e SE). O IDHM do Estado do Maranhão foi o que obteve maior variação $(34,24 \%)$ no contexto nacional e regional no período em análise. No ano de 2010, todos os estados situavam-se na faixa de médio desenvolvimento humano (Tabela 1 e Figura 3).

A Região Centro-Oeste avançou no desenvolvimento humano, pois o IDHM passou de 0,639 (médio) em 2000 para 0,757 (alto) em 2010. O Distrito Federal destaca-se desde o ano 2000, quando já apresentava o IDHM alto $(0,725)$ e, em 2010 , atingiu a faixa de muito alto $(0,824)$. Os Estados de Goiás, Mato Grosso e Mato Grosso do Sul avançaram de IDHM médio para alto no mesmo período. A Região atingiu, em 2010, o segundo maior IDHM dentre as regiões, ficando atrás apenas da Região Sudeste (Tabela 1 e Figura 3).

A melhora no desenvolvimento humano também foi observada na Região Sudeste no período de 2000 a 2010. Dentre as cinco Regiões, foi a que consignou a menor variação (13,31\%), 
partindo do IDHM 0,676 (2000) para 0,766 (2010). Dos quatro Estados da Região, apenas São Paulo manteve-se na mesma faixa (alto). Dentre as 27 UFs, São Paulo registrou a menor variação (11,54\%) no período de estudo. Os demais Estados (ES, MG e RJ) passaram do desenvolvimento humano médio para alto (Tabela 1 e Figura 3).

Assim como as demais Regiões do Brasil, a Região Sul teve melhora no IDHM, passando de 0,66 (médio) em 2000, para 0,754 (alto) em 2010. Dentre os Estados da Região, observou-se uma homogeneidade em 2000 e 2010. Paraná, Santa Catarina e Rio Grande do Sul avançaram de desenvolvimento humano médio para alto (Tabela 1 e Figura 3).

No IDHM de 2010, todos estados da Região Norte, Nordeste mais o Estado de Mato Grosso, situaram-se abaixo do IDHM do País (Figura 4).

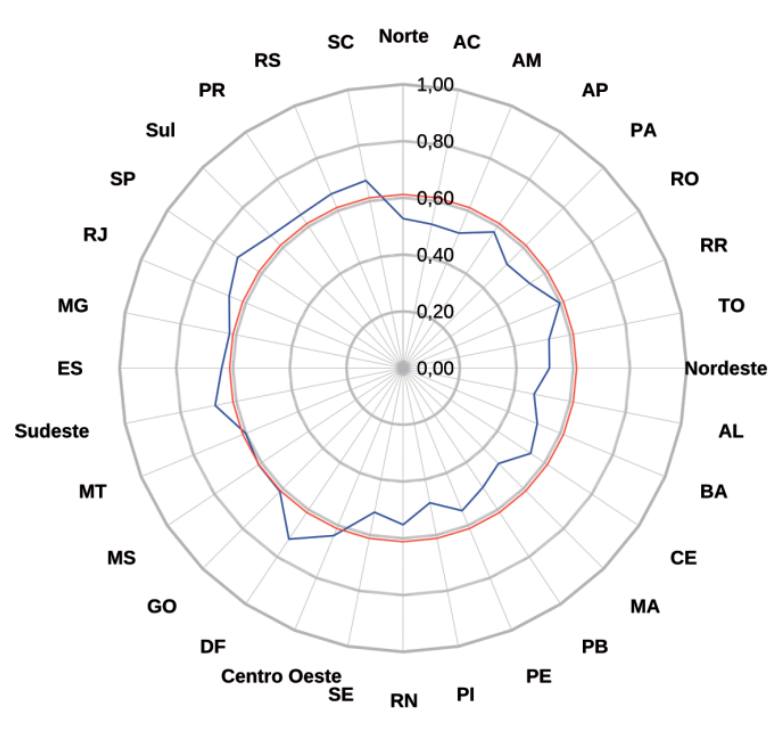

— IDHM Grandes Regiões e UF's 2000_— IDHM Brasil 2000

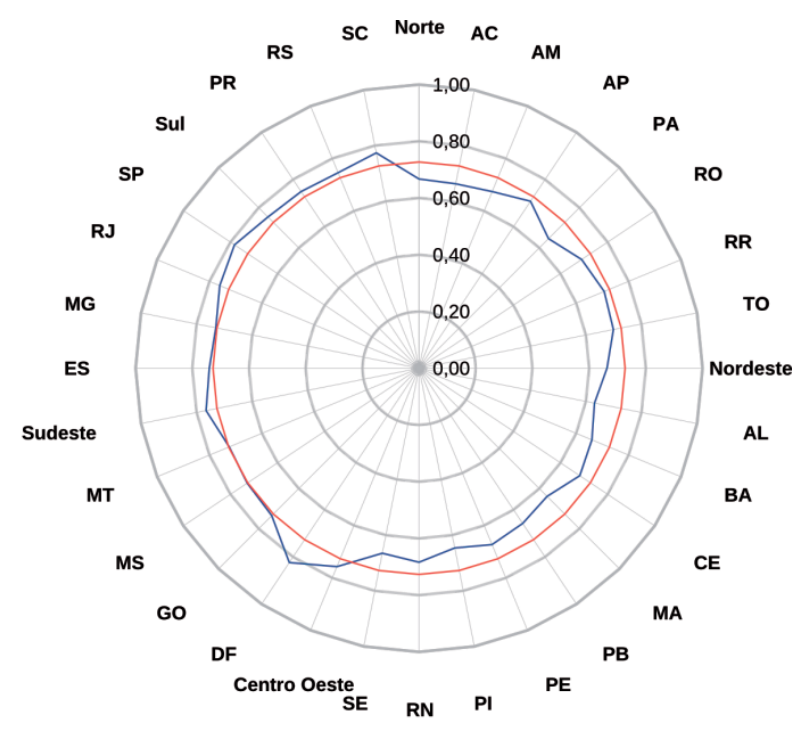

—IDHM Grandes Regiões e UF's 2010- IDHM Brasil 2010

Figura 4 - Distribuição do IDHM, segundo as Unidades da Federação, Grandes Regiões em relação ao índice do Brasil- 2000/2010

O aumento da dimensão Longevidade do IDHM indica progressos nas condições de vida e acessibilidade a serviços de saúde dos indivíduos. Outra associação presente no IDHM Longevidade é o declínio da fecundidade e da mortalidade infantil (PNUD, 2013).

\section{ANÁLISE DE CONVERGÊNCIA: BRASIL E GRANDES REGIÕES}

A média da TMI observada no Brasil e nas Grandes Regiões teve redução no período de estudo, com destaque para a Região Nordeste, onde, em 2000, a TMI era de 36,5\% e passou para 18,8\% no ano de 2010. Para essa mesma região, chama a atenção que, apesar da redução de cerca de 50\%, houve aumento do coeficiente de variação de 4,9\% para 10,5\%, sinalizando uma maior dispersão da TMI (Tabela 2).

Em 2000, das cinco Grandes Regiões, duas (Norte e Nordeste) tinham as médias do IDHM consideradas baixas, e três (Centro-Oeste, Sudeste e Sul) consideradas com médio desenvolvimento humano. No ano de 2010, as Regiões Norte e Nordeste ascenderam para um desenvolvimento humano médio e as Regiões Centro-Oeste, Sudeste e Sul obtiveram médias do IDHM consideradas altas. Houve redução no coeficiente de variação para o Brasil e Grandes Regiões. O CV praticamente manteve-se constante somente na Região Sul (Tabela 2). 
Houve convergência entre a redução das Taxas de Mortalidade Infantil e aumento do Índice de Desenvolvimento Humano Municipal, de forma não homogênea nas áreas de estudo. Nas áreas de maior desenvolvimento socioeconômico, as Taxas de Mortalidade Infantil e os índices de Desenvolvimento Humano variaram com intensidade menor, permanecendo em níveis adequados, no entanto, em regiões com piores condições de vida, houve maior variabilidade das Taxas de Mortalidade Infantil. Isso demonstra que as Taxas de Mortalidade Infantil foram bastante sensíveis às diferenças regionais. O IDHM foi menos sensível, visto que apresenta limitações intrínsecas para expressar diferenças internas, visto que é construído a partir de subíndices (longevidade, renda e educação), no entanto, de maneira geral, houve redução da TMI e consequente aumento do IDHM em todas as áreas estudadas.

Tabela 2 - Coeficiente de Variação (CV) da Taxa de Mortalidade Infantil (TMI) e do Índice de Desenvolvimento Humano Municipal (IDHM), segundo Brasil, Grande Regiões e Unidades da Federação- 2000/2010

\begin{tabular}{|c|c|c|c|c|c|}
\hline Indicadores & UFs & $\begin{array}{l}\mathrm{MI} \% \\
(2000)\end{array}$ & $\begin{array}{l}\mathrm{MI} \% \\
(2010)\end{array}$ & $\begin{array}{l}\text { IDHM } \\
(2000)\end{array}$ & $\begin{array}{l}\text { IDHM } \\
\text { (2010) }\end{array}$ \\
\hline Brasil & 27 & & & & \\
\hline Média & & 28,6 & 17,3 & 0,576 & 0,705 \\
\hline Desvio Padrão & & 8,3 & 3,8 & 0,073 & 0,049 \\
\hline CV (\%) & & 28,9 & 21,9 & 12,7 & 7,0 \\
\hline Região Norte & 7 & & & & \\
\hline Média & & 31,5 & 20,8 & 0,541 & 0,684 \\
\hline Desvio Padrão & & 4,7 & 2,4 & 0,033 & 0,024 \\
\hline CV (\%) & & 15,0 & 11,4 & 6,1 & 3,5 \\
\hline Região Nordeste & 9 & & & & \\
\hline Média & & 36,5 & 18,8 & 0,512 & 0,660 \\
\hline Desvio Padrão & & 1,8 & 2,0 & 0,030 & 0,019 \\
\hline CV (\%) & & 4,9 & 10,5 & 5,9 & 2,8 \\
\hline Região Centro-Oeste & 4 & & & & \\
\hline Média & & 22,6 & 15,8 & 0,639 & 0,753 \\
\hline Desvio Padrão & & 5,9 & 3,0 & 0,058 & 0,047 \\
\hline CV (\%) & & 26,2 & 19,2 & 9,1 & 6,3 \\
\hline Região Sudeste & 4 & & & & \\
\hline Média & & 20,4 & 13,6 & 0,658 & 0,754 \\
\hline Desvio Padrão & & 3,8 & 2,0 & 0,034 & 0,023 \\
\hline CV (\%) & & 18,5 & 14,9 & 5,2 & 3,1 \\
\hline Região Sul & 3 & & & & \\
\hline Média & & 16,8 & 11,5 & 0,663 & 0,756 \\
\hline Desvio Padrão & & 2,0 & 0,5 & 0,012 & 0,015 \\
\hline CV (\%) & & 11,9 & 3,9 & 1,8 & 2,0 \\
\hline
\end{tabular}

Fonte: Dados da pesquisa. 


\section{CONCLUSÃO}

Este estudo analisa a evolução das Taxas de Mortalidade Infantil concomitantemente com o IDHM do Brasil, Grandes Regiões e Unidades da Federação no período de 2000 a 2010. Os resultados apontam redução da TMI e aumento do IDHM em todas as áreas estudadas. Entretanto, apesar da melhora dos indicadores, eles ainda têm diferenciais quando se analisam os dados por Unidades da Federação. O desenvolvimento humano, quando comparado à mortalidade de menores de um ano, apresenta menor variabilidade, ou seja, é mais homogêneo que a mortalidade de menores de um ano.

\section{REFERÊNCIAS}

BRASIL. Ministério da Saúde. Secretaria de Vigilância em Saúde. Departamento de Vigilância de Doenças e Agravos Não Transmissíveis e Promoção da Saúde. Saúde Brasil 2014: uma análise da situação de saúde e das causas externas. Brasília: Ministério da Saúde, 2015.

FISCHER, T. K. et al. A mortalidade infantil no Brasil: série histórica entre 1994-2004 e associação com indicadores socioeconômicos em municípios de médio e grande porte. Medicina, Ribeirão Preto, SP, v. 40, n. 4, p. 559-66, out./dez. 2007.

INSTITUTO BRASILEIRO DE GEOGRAFIA E ESTATÍSTICA (IBGE). Coordenação de População e Indicadores Sociais. Síntese de indicadores sociais: uma análise das condições de vida da população brasileira: 2015. Estudos \& pesquisas. Informação demográfica e socioeconômica, Rio de Janeiro, n. 35, 2015.

INSTITUTO BRASILEIRO DE GEOGRAFIA E ESTATÍSTICA (IBGE). Tábua completa de mortalidade para o Brasil - 2015. Breve análise da evolução da mortalidade no Brasil. 2016. Disponível em: <ftp://ftp.ibge.gov.br/ Tabuas_Completas_de_Mortalidade/Tabuas_Completas_de_Mortalidade_2015/tabua_de_mortalidade_ analise.pdf>. Acesso em: 16 nov. 2016.

INSTITUTO DE PESQUISA ECONÔMICA APLICADA (IPEA). Secretaria de Planejamento e Investimentos Estratégicos Objetivos de Desenvolvimento do Milênio. Relatório Nacional de Acompanhamento. Grupo Técnico para o acompanhamento dos ODM. Brasília: Ipea: MP, SPI, 2014.

JANNUZZI, P. de M. Indicadores para diagnóstico, monitoramento e avaliação de programas sociais no Brasil. Revista do Serviço Público Brasília, Brasília, v. 56, n. 2, p. 137-60, abr./jun. 2005.

KAEMPFFER, A. R.; MEDINA, E. L. Mortalidad infantil reciente em Chile: éxitos Y desafios. Revista Chilena de Pediatría, Santiago, Chile, v. 77, n. 5, p. 492-500, out. 2006

LINS, J. G. M. G. et al. Análise espacial da evolução do índice de desenvolvimento humano nos municípios da região nordeste. Revista Economia e Desenvolvimento, Recife, PE, v. 14, n. 1, p. 81-96, jan./jun. 2015.

MEDRONHO, R. de A. et al. Epidemiologia. São Paulo: Atheneu, 2009.

POPULATION REFERENCE BUREAU (PRB). Cuadro de Datos de La Población Mundial 2014. Disponível em: <http://www.prb.org/pdf14/2014-world-population-data-sheet_spanish.pdf>. Acesso em: 16 nov. 2016.

PROGRAMA DAS NAÇÕES UNIDAS PARA O DESENVOLVIMENTO (PNUD). Desenvolvimento humano nas macrorregiões brasileiras: 2016. Brasília: PNUD; IPEA; FJP, 2016. Disponível em: <http://repositorio.ipea. gov.br/bitstream/11058/6217/1/Desenvolvimento\%20humano\%20nas\%20macrorregi\%C3\%B5es\%20 brasileiras.pdf>. Acesso em: 16 nov. 2016.

Relatório do Desenvolvimento Humano 2015. O trabalho como motor do desenvolvimento humano. 2015. Disponível em:<http://hdr.undp.org/sites/default/files/hdr_2015_report_pt.pdf>. Acesso em: 16 nov. 2016.

Índice de Desenvolvimento Humano Municipal Brasileiro 2013. Brasília: PNUD, Ipea, FJP, 2013. Disponível em: <http://www.ipea.gov.br/portal/images/stories/PDFs/130729_AtlasPNUD_2013.pdf>. Acesso em: 16 nov. 2016. 
REDE INTERAGENCIAL DE INFORMAÇÕES PARA A SAÚDE (RIPSA). Indicadores de mortalidade. Taxa de mortalidade infantil. 2012. Disponível em: <tabnet.datasus.gov.br/cgi/idb2012/c01b.htm>. Acesso em: 21 jan. 2017.

. Indicadores básicos para a saúde no Brasil: conceitos e aplicações. 2. ed. Brasília: Organização Pan-Americana da Saúde, 2008.

SCHELL, C. O. et al. Socioeconomic determinants of infant mortality: a wordwide study of 152 low, middle, and highincome countries. Scandinavian Journal of Public Health, v. 35, n. 3, p. 288-97, 2007.

SOUSA, Angelica; HILL, Kenneth; DAL POZ, Mario R. Sub-national assessment of inequality trends in neonatal and child mortality in Brazil. International Journal for Equity in Health, n. 9, 2010. Disponível em: <https://equityhealthj.biomedcentral.com/articles/10.1186/1475-9276-9-21>. Acesso em: 21 jan. 2017.

SOUSA, T. R. V.; LEITE FILHO, P. A. M. Análise por dados em painel do status de saúde no Nordeste brasileiro. Revista de Saúde Pública, São Paulo, v. 42, n. 5, p. 796-804, out. 2008.

THE WORLD BANK GROUP. Mortality rate, infant (per 1,000 live births). 2016. Disponível em: <http://data. worldbank.org/indicator/SP.DYN.IMRT.IN?view=chart>. Acesso em: 21 jan. 2017.

VICTORA, C.G. et al. Maternal and child health in Brazil: progress and challenges. Lancet, v. 377, n. 9780, p. 1863- 76, 2011.

\section{Sobre os autores:}

Paulo Cezar Rodrigues Martins: Possui graduação em Ciências Econômicas pela Faculdades Unidas Católicas de Mato Grosso - atual UCDB- (1987). É tecnologista em informações geográficas e estatísticas da Fundação Instituto Brasileiro de Geografia e Estatística. Tem experiência na área de Demografia, e Indicadores Socioeconômicos. Mestre em Saúde e Desenvolvimento na Região Centro Oeste. Atualmente é doutorando na UFMS no programa Saúde e Desenvolvimento na Região Centro Oeste. E-mail: pcrmpaulo@gmail.com

Elenir Rose Jardim Cury Pontes: Graduado em Odontologia (1987) e mestre em Saúde Coletiva (1997) pela Universidade Federal de Mato Grosso do Sul (UFMS). Doutor em Saúde Pública pela Universidade de São Paulo (2002). Professora associada da UFMS na graduação (Odontologia e Nutrição) e nos Programas de Pós-Graduação em Saúde e Desenvolvimento na Região CentroOeste e em Doenças Infecciosas e Parasitárias. Tem experiência na área de Saúde Coletiva, com ênfase em Bioestatística e Epidemiologia.E-mail: elenirpontes@uol.com.br

Leandro Tsuneki Higa: Possui graduação em Engenharia Ambiental pela Universidade Federal de Mato Grosso do Sul (2006). Mestre em Tecnologias Ambientais pela Universidade Federal de Mato Grosso do Sul. E-mail: leandro.t.higa@gmail.com 
